\title{
Unified Approach to Imidodiphosphate-Type Brønsted Acids with Tunable Confinement and Acidity
}

\author{
Sebastian A. Schwengers, Chandra Kanta De, Oleg Grossmann, Joyce A. A. Grimm, \\ Natascha R. Sadlowski, Gabriela G. Gerosa, and Benjamin List*
}

Cite This: J. Am. Chem. Soc. 2021, 143, 14835-14844

Read Online

ABSTRACT: We have designed and realized an efficient and operationally simple single-flask synthesis of imidodiphosphatebased Brønsted acids. The methodology proceeds via consecutive chloride substitutions of hexachlorobisphosphazonium salts, providing rapid access to imidodiphosphates (IDP), iminoimidodiphosphates (iIDP), and imidodiphosphorimidates (IDPi). These privileged acid catalysts feature a broad acidity range $\left(\mathrm{p} K_{\mathrm{a}}\right.$ from $\sim 11$ to $<2$ in $\mathrm{MeCN}$ ) and a readily tunable confined active site. Our approach enables access to previously elusive catalyst scaffolds with particularly high structural confinement, one of which catalyzes the first highly enantioselective (>95:5 er) sulfoxidation of methyl $n$-propyl sulfide. Furthermore, the methodology delivers a novel, rationally designed

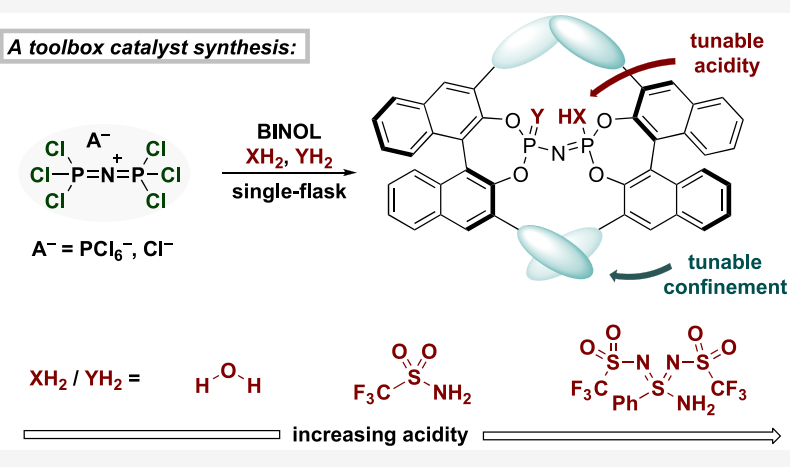
super acidic catalyst motif, imidodiphosphorbis(iminosulfonylimino)imidate (IDPii), the extreme reactivity of which exceeds commonly employed super-Brønsted acids, such as trifluoromethanesulfonic acid. The unique reactivity of one such IDPii catalyst has been demonstrated in the first $\alpha$-methylation of a silyl ketene acetal with methanol as the electrophilic alkylating reagent.

\section{INTRODUCTION}

Acid catalysis is arguably the single most general approach to catalysis there is. It enables the activation of diverse and inherently distinct substrate classes, which, at least in principle, as a necessary and sufficient condition, only require electron density and as such, the potential to catalytically activate the vast majority of all chemical materials exists. It is therefore perhaps not surprising that acidic catalysts have become indispensable tools for chemical synthesis as well as an enabling technology for multimillion-ton-scale productions. ${ }^{1}$ During the last two decades, organic Brønsted acids have enriched the arsenal of asymmetric catalysis, initially in bifunctional catalysts such as proline or BINOL-derived phosphoric acids (CPA), ${ }^{2,3}$ and lately also in more purely acidic motifs. ${ }^{4}$ In this context, we have generalized the underlying principle of asymmetric Brønsted acid catalysis, in which protons act as the activating principle while chiral, enantiopure anions enable enantiodifferentiation, toward asymmetric counteranion directed catalysis (ACDC), including all types of cationic activation principles. ${ }^{5}$ The high versatility of Brønsted acids inspired the development of ever more acidic catalysts to overcome intrinsic reactivity barriers of weakly basic substrates. ${ }^{6}$ However, the highly selective conversion of small and constitutionally unbiased substrates has long remained challenging due to the rather open active site of most Brønsted acid catalysts and the resulting conformational freedom of protonated reactive intermediates and transition states. To overcome these limitations, our group has conceptualized, designed, and established confined acids, the corresponding bases of which possess highly compact anionic active sites. Such counteranions are suggested to formally bind and stabilize cationic transition states of reactions involving small, unfunctionalized substrates. In 2012, we introduced the first generation of such catalysts, dimeric $C_{2}$-symmetric imidodiphosphates (IDP). ${ }^{7}$ With their four 3,3'-substituents on the binaphthyl backbone, these catalysts provide a well-defined and very tight microenvironment. IDP catalysts have consequently emerged as powerful and versatile catalyst scaffolds, somewhat resembling enzymatic substrate recognition. Due to the diversity of the substituted and modified BINOL backbone, a broad range of distinct cavities, displaying designable substrate-class recognition, are readily accessible and enable highly stereoselective transformations of previously elusive substrates. ${ }^{8}$ However, whereas IDPs $\left(\mathrm{p} K_{\mathrm{a}} \approx 11\right.$ in $\left.\mathrm{MeCN}\right)$ are significantly stronger acids than

Received: July 7, 2021

Published: September 3, 2021

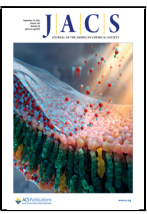


A A privileged scaffold in asymmetric catalysis:
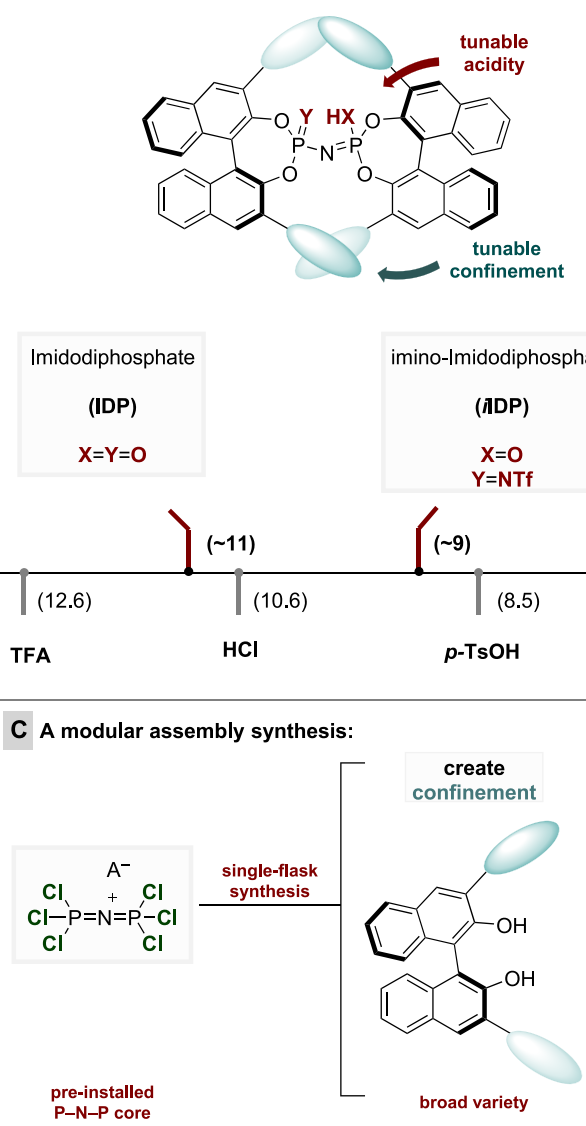

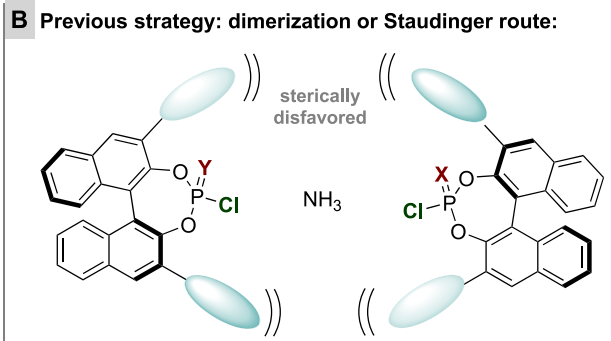

$x$ multi-step syntheses

$x$ limited access (steric repulsion)

$x$ challenging formation

$(\sim 3)$

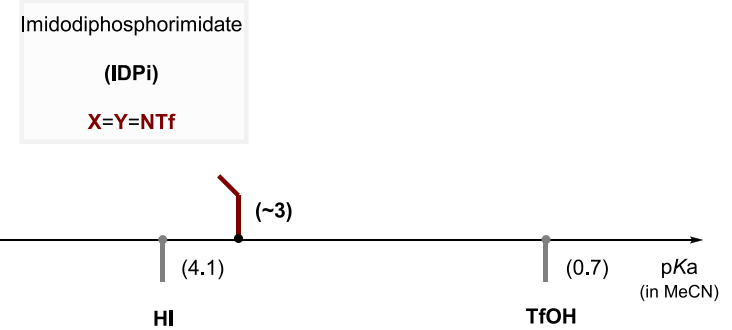

$\left(4-\mathrm{NO}_{2} \mathrm{C}_{6} \mathrm{H}_{4}\right) \mathrm{SO}_{3} \mathrm{H}$

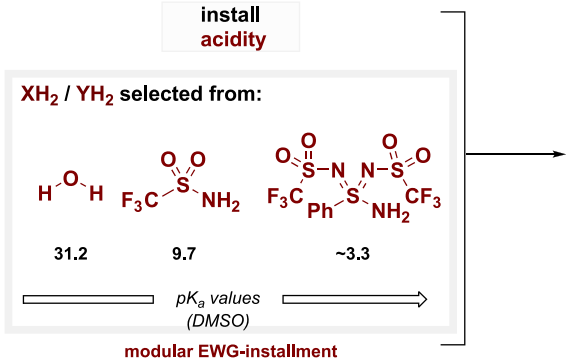

modular EWG-installment

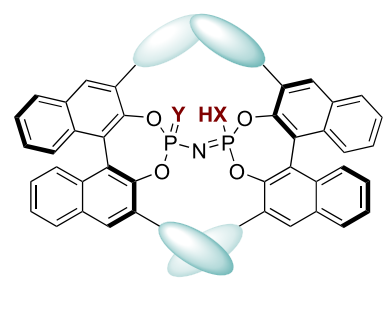

new access to high acidity \& confinement

Figure 1. Synthesis of imidodiphosphoryl-derived Brønsted acids.

chiral phosphoric acids (CPAs, $\mathrm{p} K_{\mathrm{a}} \approx 13$ in $\mathrm{MeCN}$ ), their acidity is still moderate, limiting their applicability to relatively basic substrates such as imines, enol ethers, and certain carbonyl compounds. The replacement of oxygen atoms with one or two NTf groups led to the development of iIDPs and IDPis, respectively, comprising high and tunable acidities $\left(\mathrm{p} K_{\mathrm{a}}\right.$ $\leq 2-9$ in $\mathrm{MeCN}$, Figure $1 \mathrm{~A}$ ), in combination with excellent stereoinduction from the enantiopure counteranion. ${ }^{9-11}$ IDPis have also found utility as precatalysts for powerful and userfriendly silylium-based Lewis acid catalysis and have enabled extremely challenging transformations. ${ }^{12}$ The combination of modular acidity and tunable confinement has led to unprecedented and unusual transformations in organocatalysis, such as an organocatalytic olefin activation, ${ }^{13}$ the selective monoaldolization of acetaldehyde enolates, ${ }^{14}$ a widely applicable Prins cyclization, ${ }^{9}$ or a challenging Mukaiyama aldol reaction with sub-ppm catalyst loadings ${ }^{15}$ and the handling of nonclassical carbocations. ${ }^{16}$

Our previous approach to imidodiphosphoryl-type scaffolds relied either on the dimerization of two phosphoryl halide moieties (Figure 1B) in the presence of ammonia or a surrogate for the synthesis of IDPs and IDPis or on a Staudinger approach of a phosphoryl azide with an $\mathrm{N}$-sulfonyl phosphoramidite to furnish iIDPs. ${ }^{7,910}$ The synthesis of IDP and $i \mathrm{IDP}$ catalysts required the preformation and isolation of the corresponding monomeric phosphoryl units prior to the dimerization/Staudinger approach for each catalyst synthesis. Additionally, $\mathrm{N}$-sulfonyl substituent modifications for the iIDP and IDPi motif rely on the preparation and isolation of $\mathrm{N}$ - sulfonylphosphorimidoyl trichloride or $\mathrm{N}$-sulfonyl phosphoramidites, respectively, for every core modification, resulting in a time-consuming catalyst library establishment. ${ }^{17}$ Importantly, the dimerization process is strongly influenced by steric properties of the $3,3^{\prime}$-substituents on the BINOL moiety and occasionally provides unsatisfactory yields, proceeds under harsh reaction conditions, or requires prolonged reaction times. BINOLs with highly sterically demanding substituents often do not furnish the desired imidodiphosphoryl motif due to steric repulsion in the dimerization process (Figure 1B). We became highly motivated to address this problem since we are particularly interested in catalysts possessing extreme confinement in combination with extreme acidities, which we deem a requirement toward handling very small and nonactivated substrates. ${ }^{11}$ We now report a new, unified, general, and userfriendly synthetic strategy toward imidodiphosphate-type motifs. A particular focus is given to previously elusive catalyst scaffolds and toward the development of even more acidic imidodiphosphoryl-based Brønsted acids, which overcomes remaining reactivity barriers and facilitates the development of novel transformations within the ACDC framework. ${ }^{5,18}$

\section{RESULTS AND DISCUSSION}

To circumvent the limitations of our earlier developed methods, and to establish a more efficient, straightforward, and operationally simple catalyst synthesis, we envisioned utilizing hexachlorobisphosphazonium hexachlorophosphate (HCPP), initially reported by Becke-Goehring, ${ }^{19,20}$ as a platform molecule for the synthesis of dimeric imidodiphos- 
Scheme 1. IDP Synthesis and Reaction Scope
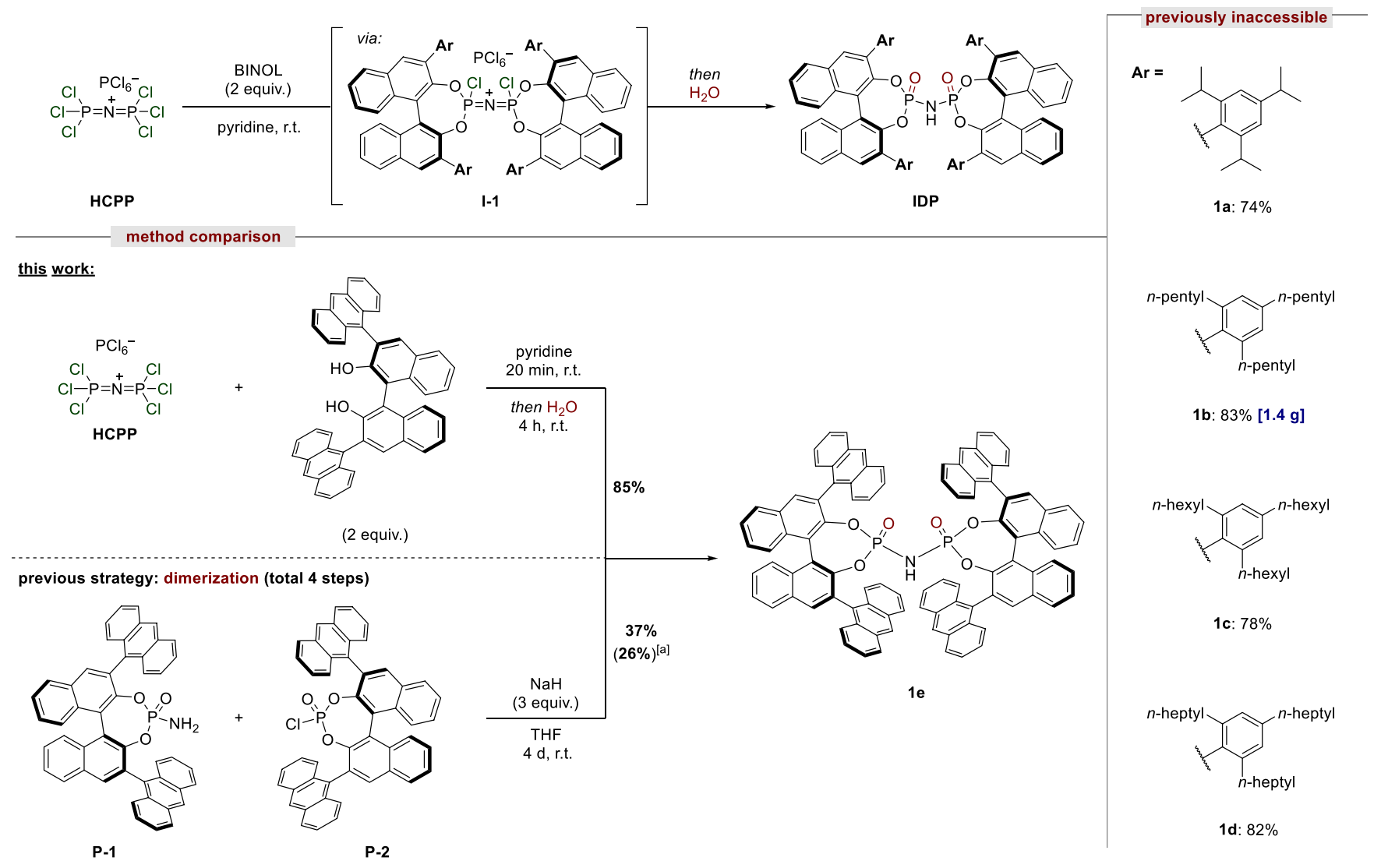

$1 \mathrm{~b}: 83 \%[1.4 \mathrm{~g}]$

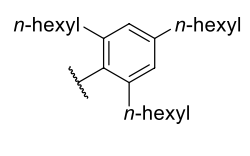

1c: $78 \%$

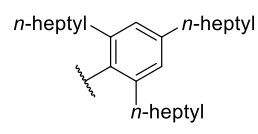

1d: $82 \%$

${ }^{a}$ Yield over four steps from BINOL.

phoryl-derived Brønsted acids (Figure 1C). Using HCPP as the starting material would bear the following advantages: (a) the $\mathrm{P}-\mathrm{N}-\mathrm{P}$ core is already preinstalled, avoiding inefficient dimerizations by mitigating steric repulsion during the dimerizing event; (b) intermediate I-1, which we expected to form upon treating HCPP with two BINOLs (Scheme 1) would be functionalizable by simple chloride substitution with suitable electron-withdrawing groups (EWGs), e.g., sulfonamides; (c) all previously mentioned imidodiphosphoryl-type Brønsted acids would be accessible from the same common intermediate I-1; (d) HCPP is readily available in a single step on decagram scales, stable, and would allow simplified largescale catalyst syntheses and ideally furnishes the desired products in high yields with single product isolation and simplified purification procedures.

We started exploring the reactivity of HCPP by focusing on the synthesis of imidodiphosphates (Scheme 1). We found that, in pyridine, a rapid reaction of HCPP with different BINOLs occurs, resulting in the formation of intermediates I1, which upon addition of water readily provides the desired IDP products. Remarkably, IDPs $\mathbf{1 a}-\mathbf{d}$, which were inaccessible with our previously established method, likely due to high steric repulsion within the dimerization, are now readily available. Furthermore, we compared the efficiency of our new methodology to the previously established dimerization approach. Phosphoryl amide P-1 and phosphoryl chloride P2 were independently synthesized and reacted with sodium hydride to furnish the desired IDP 1e after 4 days in $37 \%$ yield. ${ }^{7}$ In contrast, our new methodology provides IDP 1e from the corresponding BINOL in less than $5 \mathrm{~h}$ and in $85 \%$ yield, which only requires a single and simplified purification step.

With the newly established procedure toward IDPs, in which salt I-1 was found to be the key intermediate, we envisioned that substituting a chloride of I-1 with trifluoromethanesulfonamide $\left(\mathrm{TfNH}_{2}\right)$, followed by hydrolysis, should furnish the corresponding iIDP motif. Owing to its enhanced acidity but relatively complicated previous synthesis, an expeditious route to this catalyst class is particularly attractive. Indeed, due to the highly electrophilic character of intermediate I-1, a rapid substitution of chloride with $\mathrm{TfNH}_{2}$ occurs within minutes, resulting in the formation of neutral intermediates I-2, which upon hydrolysis with water afforded the desired iIDP products (Scheme 2). Our modular approach enables previously unexplored BINOL and sulfonamide combinations, smoothly providing $i$ IDPs $\mathbf{2 a - d}$ in good yields, following a single-flask procedure and a simplified purification. Once again, the TRIPBINOL-derived product iIDP 2 a was previously inaccessible and is now readily available using the new procedure. Furthermore, various $N$-sulfonyl groups can now be easily introduced by simple chloride substitution of intermediate I-1 with the sulfonamide of choice, as shown with iIDPs $\mathbf{2} \mathbf{b}$ and 2c. The structure of iIDP $2 \mathrm{~d}$ has been investigated by X-ray crystallography, illustrating the bifunctional active center coordinated to two $\mathrm{H}_{2} \mathrm{O}$ molecules in a structurally confined cavity.

We also investigated the utility of our new approach toward the more acidic IDPi catalyst class (Scheme 3). As hoped, the final chloride substitution of intermediate I-2 indeed occurs under elevated temperatures and slightly modified reaction 
Scheme 2. iIDP Synthesis, Reaction Scope, and Single-Crystal Structure of $2 \mathrm{~d} \cdot 2 \mathrm{H}_{2} \mathrm{O}$

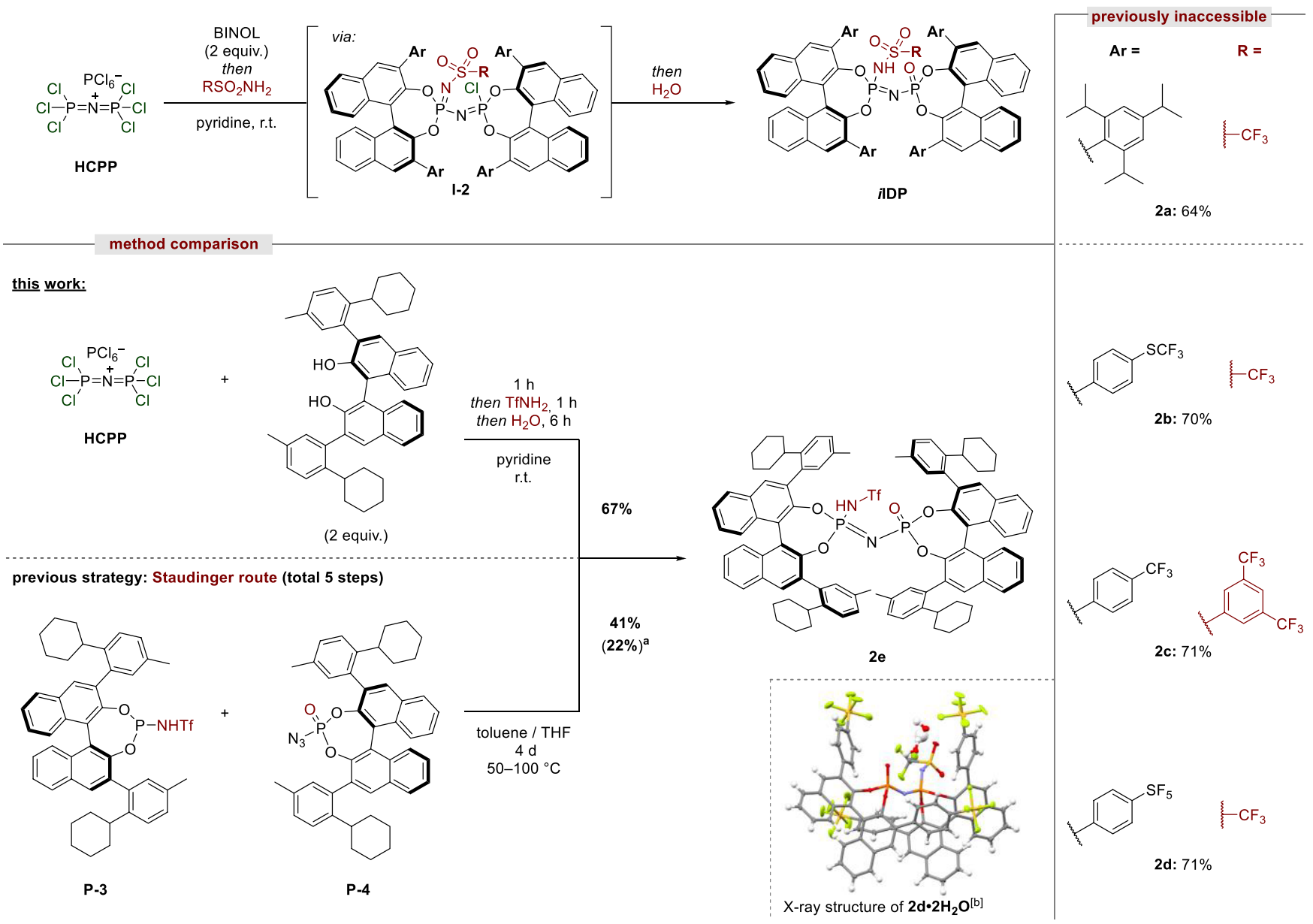

${ }^{a}$ Yield over five steps from BINOL. ${ }^{b}$ Two disordered $\mathrm{CH}_{2} \mathrm{Cl}_{2}$ molecules are omitted for clarity.

conditions (replacing pyridine with $\mathrm{NEt}_{3}$ and using toluene as solvent). Highly confined IDPis $\mathbf{3} \mathbf{a}, \mathbf{b}$, which were previously elusive following our in situ dimerization strategy are now readily accessible, thus expanding the repertoire of novel, structurally confined motifs of this catalyst class. Furthermore, a simple chloride substitution with different sulfonamides, as illustrated with product $3 \mathrm{c}$, allows a rapid sulfonyl group modification. Following our previous route, access to such IDPi motifs would require a prior synthesis of the corresponding $\mathrm{N}$-sulfonylphosphorimidoyl trichloride. ${ }^{17}$ Although the yields were only moderate, unreacted intermediates, such as I-2, for the synthesis of IDPi 3a are isolable by simple flash column chromatography or directly furnish the corresponding iIDP upon hydrolysis. We compared the previous dimerization strategy with our new method for the synthesis of IDPi 3d. Again, the new methodology affords IDPi $3 \mathbf{d}$ in a shorter reaction time and improved yield.

With the established new access to novel and unexplored imidodiphosphates comprising unprecedented structural confinement, we turned our attention to the activation of previously elusive small substrates in asymmetric catalysis. We chose methyl $n$-propyl sulfide 4 as a model substrate for the IDP-catalyzed asymmetric sulfoxidation, ${ }^{21}$ in which our previous benchmark IDP catalyst 1f furnished an unsatisfactory enantiomeric ratio of $91.5: 8.5$ of the sulfoxide 5. Remarkably, IDP 1c was found to be a superior catalyst for this particularly challenging substrate and delivered the product in 95:5 er
(Scheme 4). It should be noted that this is by far the highest enantioselectivity ever obtained with this particular substrate via any type of catalytic sulfoxidation. ${ }^{22}$ Such results confirm the importance of having an efficient methodology available to access novel and highly confined catalysts, which are crucial to control structurally unbiased substrates in asymmetric catalysis.

Toward Superacidity in Asymmetric CounteranionDirected Catalysis. The formation, stabilization, and utilization of carbocationic intermediates has been extensively studied in academic research and is frequently applied in a technical context, e.g., in Koch-Haaf and Friedel-Crafts reactions. ${ }^{23}$ These transformations usually require strong mineral acids, such as $\mathrm{H}_{2} \mathrm{SO}_{4}$ or $\mathrm{TfOH}$, or strong Lewis acids to dictate the desired carbocation formation upon protonation of olefins or alcohols, whereas the stabilization of carbocationic intermediates by weakly basic counteranions is crucial to prevent undesired side reactions. ${ }^{24}$

A general approach to increase the acidity of Brønsted acids relies on the installment of electron-withdrawing groups into the existing catalyst scaffold. ${ }^{6 \mathrm{~b}}$ Trifluoromethylsulfonyl groups represent one of the strongest and presumably most wellinvestigated electron-withdrawing group, due to its nonoxidizing properties and inherent stability. ${ }^{25}$ Yagupolskii et al. successfully increased the electron-withdrawing nature of trifluoromethylsulfonyl groups by replacing the corresponding Lewis basic oxygen atoms with additional trifluoromethylsulfonylimino units (Yagupolskii principle). ${ }^{26}$ This acidification 
Scheme 3. IDPi Synthesis and Reaction Scope

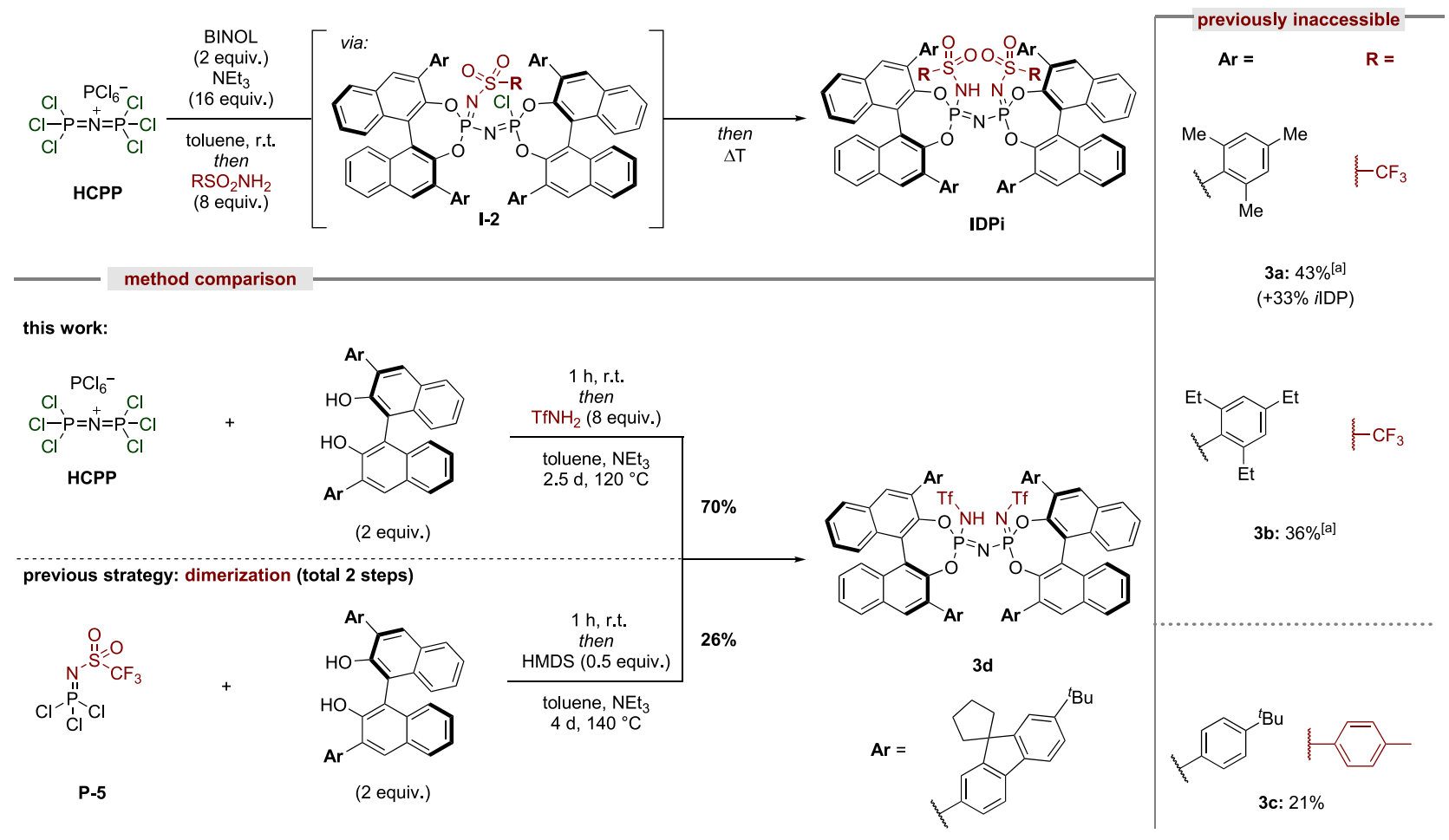

${ }^{a}$ 4-DMAP ( 9 mol \%) was added to accelerate the final chloride substitution.

Scheme 4. Catalytic Asymmetric Sulfoxidation of Methyl nPropyl Sulfide

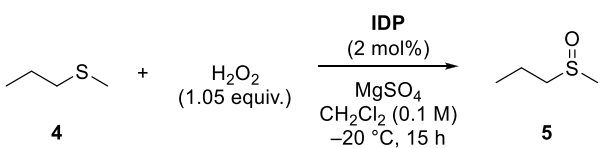

catalyst evaluation

IDP
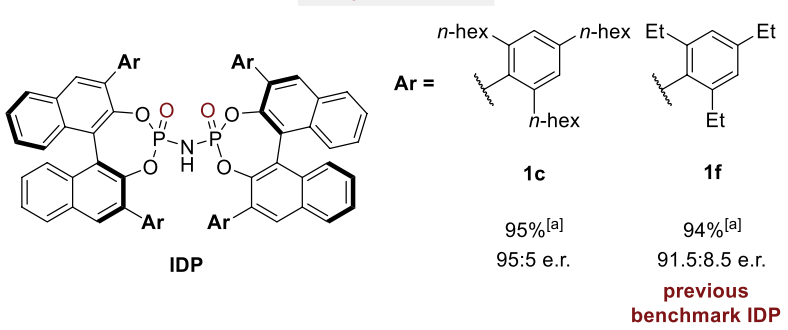

${ }^{a}$ Yields were determined by ${ }^{1} \mathrm{H}$ NMR spectroscopy with dimethyl sulfone as internal standard.

effect tremendously increases the acidity of $\mathrm{CF}_{3} \mathrm{SO}_{3} \mathrm{H}(\mathrm{TfOH}$, $\mathrm{pK}_{\mathrm{a}}=-11.4$ in DCE) to $\mathrm{CF}_{3} \mathrm{~S}(\mathrm{NTf})_{2} \mathrm{OH}\left(\mathrm{p} K_{\mathrm{a}}=-18\right.$ (estimated $\mathrm{pK}_{\mathrm{a}}$ in DCE)). ${ }^{27}$ Analogously, the replacement of Lewis basic $=\mathrm{O}$ moieties of aryl sulfonamides with $=\mathrm{NTf}$ groups increases the acidity of $\left(4-\mathrm{MeC}_{6} \mathrm{H}_{4}\right) \mathrm{SO}_{2} \mathrm{NH}_{2}(\mathrm{pK}$ 16.3 in DMSO) toward $\left(4-\mathrm{MeC}_{6} \mathrm{H}_{4}\right) \mathrm{S}(\mathrm{NTf})_{2} \mathrm{NH}_{2}\left(\mathrm{p} K_{\mathrm{a}}=3.3\right.$ in DMSO), thus enhancing the acidity by $13 \mathrm{p} K_{\mathrm{a}}$ units and exceeding the electron-withdrawing property of the commonly employed $\mathrm{TfNH}_{2}$ group $\left(\mathrm{pK}_{\mathrm{a}}=9.7\right.$ in DMSO $)$ by approximately $6 \mathrm{p} K_{\mathrm{a}}$ units.

Notably, the utilization of $\mathrm{PhS}(\mathrm{NTf})_{2} \mathrm{NH}_{2}$ (6), as the EWG substituent not only enhances the acidity but also simultaneously installs another structural element, in addition to the 3,3'-BINOL substituents, allowing a more flexible and modular implementation of confinement. $\mathrm{PhS}(\mathrm{NTf})_{2} \mathrm{NH}_{2}$ (6) was synthesized based on a modified approach reported by Yagupolskii et al. (see Supporting Information for further information) and has been further investigated in this work (Scheme 5)..$^{30}$

With the new design and reagent in hand, we evaluated the synthesis of new imidodiphosphorbis(iminosulfonylimino)imidate, IDPii, following the previously described stepwise chloride substitution as shown for the synthesis of IDPis (Scheme 3). Unfortunately, the reaction of sulfonamide 6 with intermediate I-1 (Scheme 1) proceeded slugglishly and only yielded intermediate I-3, which upon hydrolysis afforded the corresponding non- $C_{2}$-symmetric iminoimidodiphosphate in poor yields. The desired $C_{2}$-symmetric product, bearing two iminosulfonyl units derived from 6, analogously to IDPis was, unfortunately, not accessible via this route. This observation can be explained with the weak nucleophilicity of sulfonamide 6, hampering the desired chloride substitution of I-1 and I-3.

To overcome the intrinsic barrier of reacting weakly nucleophilic sulfonamide 6 with intermediate I-3, showing diminished electrophilic properties, we changed our synthetic strategy. We assumed that the direct reaction of sulfonamide 6 with HCPP, exploiting the immense electrophilic character of this reagent, followed by the BINOL installation event would be more effective. To our delight, we observed the desired transformation of HCPP with sulfonamide 6, liberating $\mathrm{HCl}$ gas to form I-4, without the requirement of a base. Unexpectedly, the corresponding $\mathrm{PCl}_{6}{ }^{-}$counteranion also reacted with sulfonamide $\mathbf{6}$ to afford phenylbis(trifluoromethylsulfonylimino)phosphorimidoyl trichloride as undesired side product, which would likely interfere in the BINOL installation step (see Supporting Information for further information). We therefore replaced the $\mathrm{PCl}_{6}{ }^{-}$ counteranion of HCPP with a chloride counteranion, following 
Scheme 5. Rational Design and Development of a More Acidic Imidodiphosphazene Catalyst by EWG Replacement

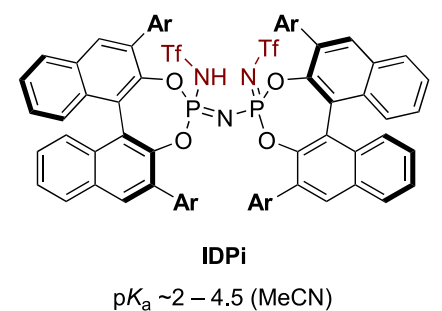

IDPii development
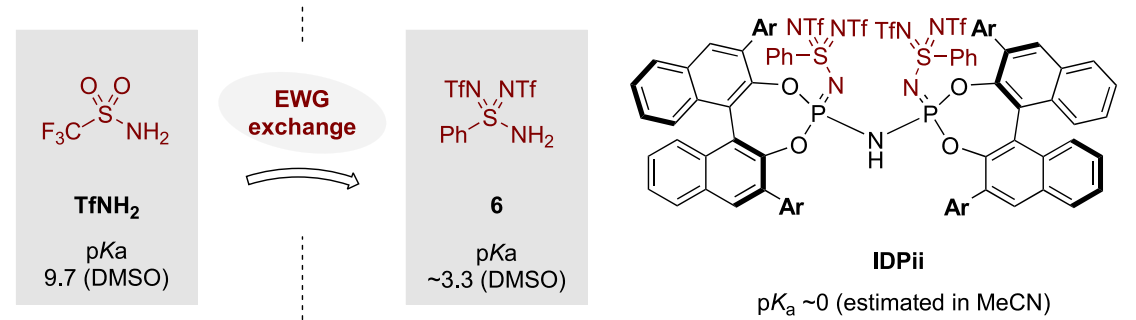

$\mathrm{p} K_{\mathrm{a}} \sim 0$ (estimated in $\mathrm{MeCN}$ )

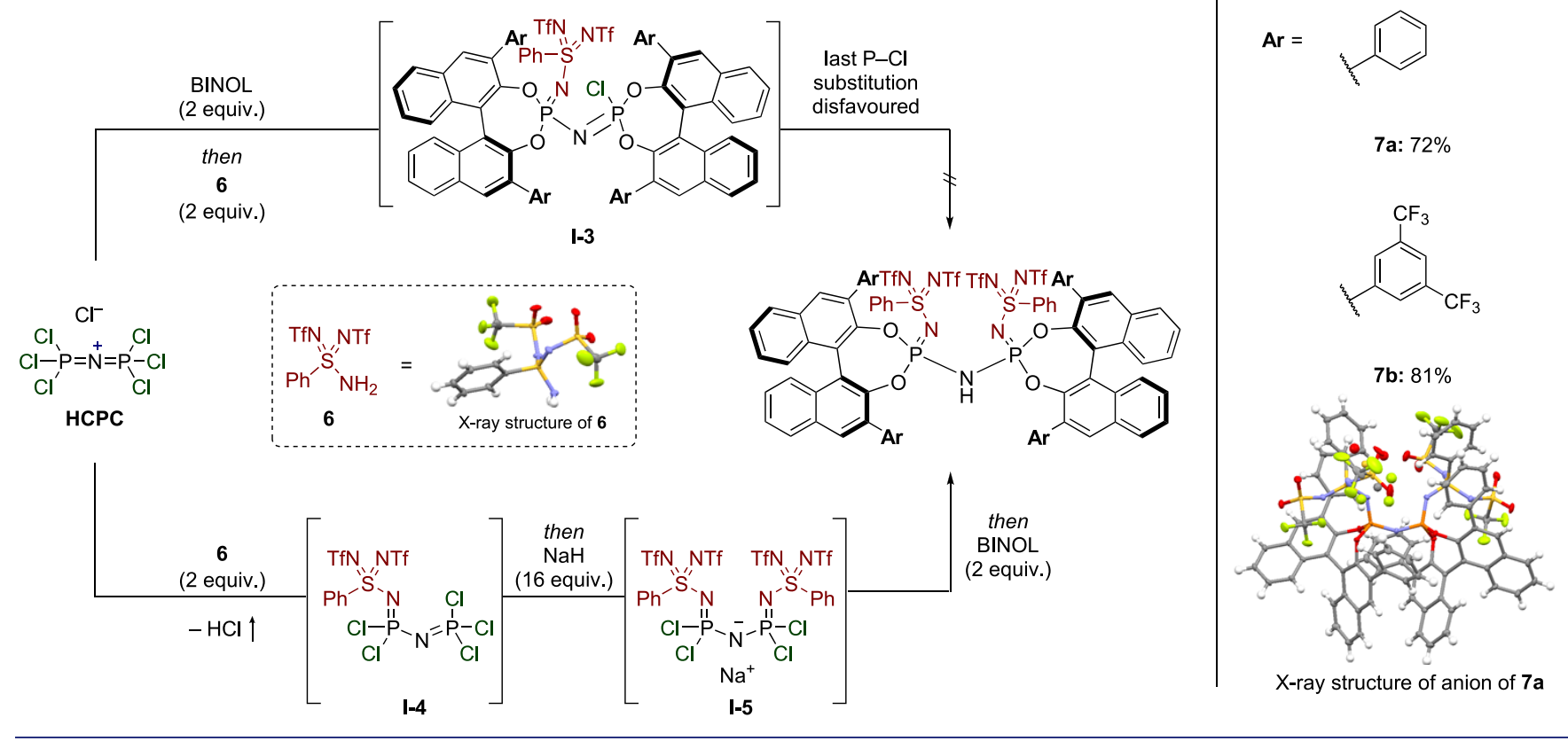

aryl modification

Manners' one-step procedure, to afford hexachlorobisphosphazonium chloride (HCPC). ${ }^{20 a}$

As expected, HCPC reacted smoothly with sulfonamide 6 to quantitatively form I-4 at room temperature within $30 \mathrm{~min}$, presenting an ideal intermediate for our desired catalyst motifs. The reaction of I-4 with another equivalent of sulfonamide $\mathbf{6}$ in the presence of sodium hydride or an organic base such as triethylamine afforded sodium bis(trifluoromethylsulfonylimino)tetrachloridophosphazenate I-5, in which both sulfonamides are installed into the imidodiphosphate scaffold. We found suitable reaction conditions, in which intermediates I-4 and I-5 are formed in situ and reacted upon addition of BINOL toward the desired catalyst motif IDPii, in a single-flask procedure, providing $7 \mathbf{a}$ and $7 \mathbf{b}$ in good yields. These catalysts were rapidly acidified, either by dissolving the corresponding salts in dichloromethane and emulsifying with aqueous $\mathrm{HCl}$ or by passing a catalyst solution through Dowex 50W-X8. It should be noted that the dimerization strategy for phenylbis(trifluoromethylsulfonylimino)phosphorimidoyl trichloride with BINOL and hexamethyldisilazane (HMDS) or ammonia yielded the desired dimer $7 \mathbf{a}$ in traces ( $6 \%$ on a large scale), whereas the formation of $\mathbf{7 b}$ was not observable. This result underlines the applicability of hexachlorobisphosphazonium salt as a building block to rationally design and successfully enhance the repertoire of imidodiphosphoryl scaffolds, which might turn out to be superior catalysts or interesting ligands for transition-metal catalysis. IDPii $7 \mathbf{a}$ was further characterized by X-ray crystallography.

With these novel catalysts in hand, we aimed toward a reactivity comparison of IDPi and IDPii, applying the same phenyl-derived BINOL substituents to evaluate the acidifying effect of our new core modification (Scheme 6). In light of recent ${ }^{29} \mathrm{Si}$ NMR studies from Oestreich and our group in combination with Gutmann-Beckett studies, ${ }^{31}$ we focused on the quantification of Lewis acidities of IDPi $3 \mathbf{e}$ and IDPii $7 \mathbf{a}$, which rapidly react with allyltrimethylsilane to furnish the corresponding Lewis acidic silylated imidodiphosphazene catalysts. ${ }^{32}$ It should be noted, that IDP and iIDP were not included in our studies due to inefficient catalytic activity as Lewis acids. As expected, our new catalyst motif IDPii 7a shows a much higher ${ }^{29} \mathrm{Si}$ chemical shift, in direct comparison to that of IDPi 3e, suggesting a significantly enhanced Lewis acidity. $^{24,33}$ Interestingly, IDPii $7 \mathbf{a}$ exceeds the chemical shift of trimethylsilyl triflate (TMSOTf) and bis(trifluoromethylsulfonyl)imide (TMSNTf ${ }_{2}$ ), which are commonly employed superacids in organic synthesis. In agreement with our experience of IDPi catalysis, trimethylsilylated IDPi 3e represents a stronger Lewis acid in comparison to TMSOTf but remains a significantly weaker Lewis acid than TMSNTf $_{2}$. The same reactivity trend has been observed in our GutmannBeckett study, in which IDPii 7a resulted in a triethylphosphine oxide shift of $\Delta \delta=39.3 \mathrm{ppm}$, whereas the utilization of IDPi 3e leads to a shift of $\Delta \delta=24.0 \mathrm{ppm}$, supporting our 
Scheme $6 .{ }^{29} \mathrm{Si}$ NMR and Gutmann-Beckett studies to quantify Lewis acidities

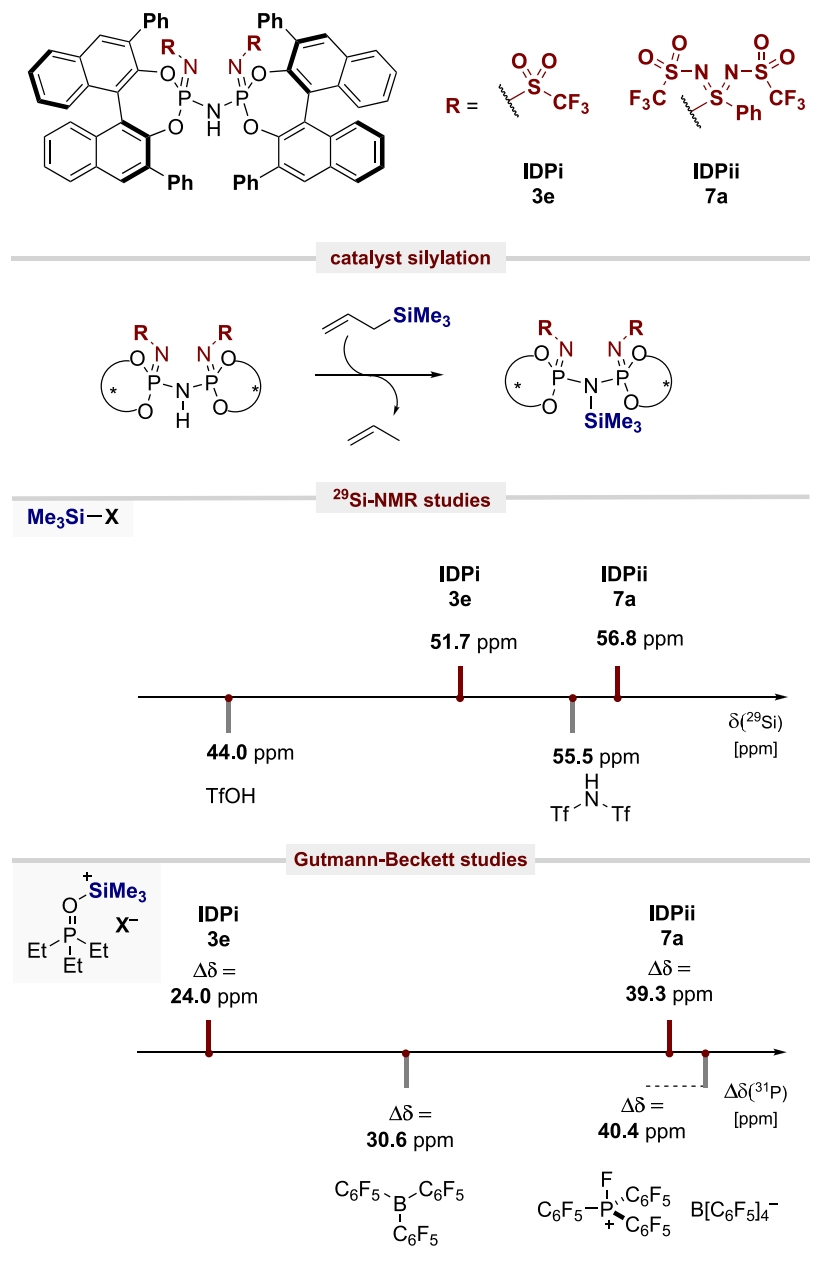

hypothesis of an increased Lewis acidity of IDPii to the analogous IDPi (see Supporting Information).

Interestingly, our Gutmann-Beckett study indicates a Lewis acidity of IDPii $7 \mathbf{a}$ that is similar to the extremely Lewis acidic fluorophosphonium tetrakis(pentafluorophenyl)borate salt, initially reported by Stephan et al., ${ }^{34}$ which has been utilized for various challenging transformations proceeding via the formation of carbocationic intermediates. ${ }^{35}$ However, these extremely Lewis acidic catalysts often require strictly inert reaction conditions to prevent catalyst degradation, especially due to hydrolysis pathways in the presence of nucleophilic and protic impurities, such as water or alcohols. In contrast, our catalyst motifs possess the advantage of extreme Lewis acidity, without the requirement of inert reaction conditions, due to the catalytic deprotosilylation cycle, in the presence of sacrificial silylating reagent. This property led to the hypothesis that we might be able to convert methanol-a normally incompatible nucleophile for many strong Lewis acids and transition-metal catalysts-into a potent electrophile. We reasoned that methanol (8) should first undergo a deprotosilylation cycle in the presence of a silylating agent, such as trimethylsilyl ketene acetal 9, to afford trimethylsilyl methyl ether 11 in situ, which in return should still be Lewis basic enough to react with another equivalent of trimethylsilylated IDPii to form the corresponding bis(trimethylsilyl)methoxonium salt 12. Analogously to Meerwein salts, ion pair 12 was envisioned to represent a powerful methylating agent, which should readily react with the nucleophilic silyl ketene acetal 9 to furnish methyl pivalate $\mathbf{1 0}$ as the final product (Scheme 7).

Scheme 7. Initial Catalyst Screening for the $\alpha$-Methylation of a Silyl Ketene Acetal with Methanol
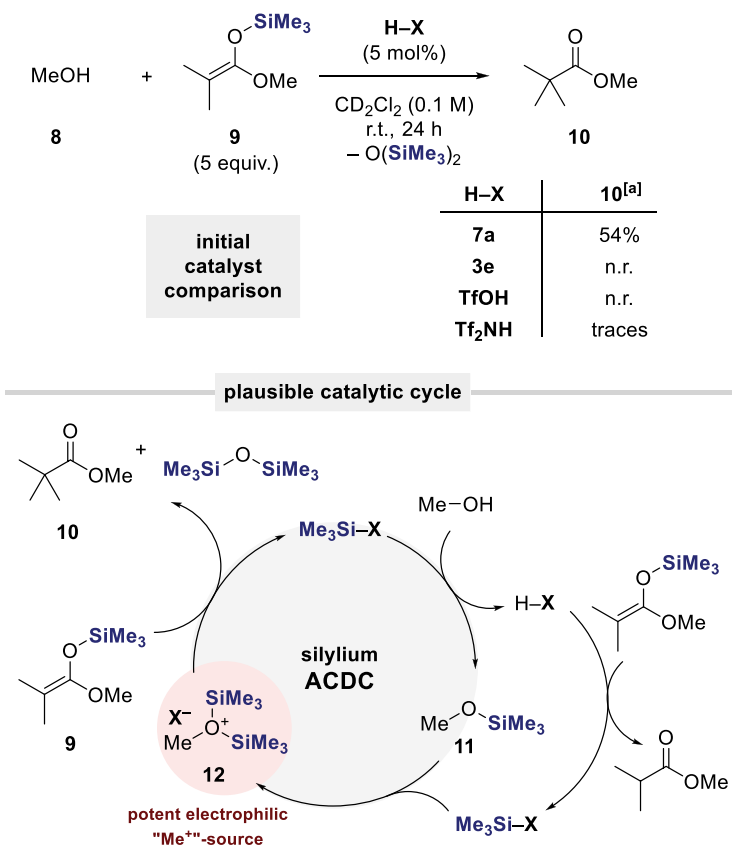

${ }^{a}$ Yields were determined by ${ }^{1} \mathrm{H}$ NMR spectroscopy with mesitylene as internal standard.

Remarkably, a comparison between $\mathrm{TfOH}, \mathrm{Tf}_{2} \mathrm{NH}$, and our IDPi and IDPii catalysts revealed that the desired transformation only proceeded with our new IDPii catalyst class, whereas the other three catalysts did not engage in the desired transformation. Their insufficient reactivity most likely results from the weaker Lewis acidity of these catalysts, consistent with our Lewis acidity measurements. Our newly designed transformation from the in situ generation of the highly potent and electrophilic methylating reagent $\mathbf{1 2}$ avoids the utilization of commonly employed toxic alkylating reagents such as dimethyl sulfate or methyl iodide. The asymmetric $\alpha$-alkylation of silyl ketene acetals and the expansion toward various silylderived nucleophiles to develop a general asymmetric dehydroxyfunctionalization strategy is currently in investigation in our laboratory and will be communicated independently.

\section{CONCLUSION}

The imidodiphosphoryl scaffold represents a highly versatile platform to design Brønsted acids, merging enzyme-like substrate recognition with modular acidities that enable several, perhaps surprising and unique, reactions in asymmetric catalysis over the past years. We have revealed a new userfriendly synthesis of imidodiphosphoryl-based catalysts, in which a hexachlorobisphosphazonium salt serves as a building block and selectively reacts with chosen nucleophiles based on a toolbox principle. This methodology, which proceeds via common key intermediates, provides a fast and highly efficient access to privileged Brønsted acids, such as IDP, iIDP, and IDPi, comprising unique and, most notably, previously 
inaccessible confinement. In fact, these catalysts were conceptually designed to provide superior enantiocontrol of small and structurally unbiased substrates, as illustrated in the first highly enantioselective sulfoxidation of methyl propyl sulfide.

Furthermore, this novel modular assembly synthesis allows the implementation of new strongly acidifying sulfonamides into the imidodiphosphoryl scaffold, empowering the conceptualization and development of an extremely reactive Brønsted acid, IDPii. Analytical and experimental studies show, under silylium Lewis acid conditions, a significantly enhanced catalytic performance for the IDPii, which overcomes the reactivity of commonly employed super-Brønsted acids, such as $\mathrm{TfOH}$ and $\mathrm{Tf}_{2} \mathrm{NH}$. The superior reactivity enables the realization of a new $\alpha$-alkylation of a silyl ketene acetal-utilizing methanol as electrophilic methyl surrogateand expands the repertoire of useful $\alpha$-alkylation strategies of carbonyl compounds. We anticipate that our methodology provides a new foundation toward future developments of novel imidodiphosphoryl-type catalysts, leading to efficient asymmetric transformation in the field of asymmetric organocatalysis or as ligands in transition-metal catalysis.

\section{ASSOCIATED CONTENT}

\section{SI Supporting Information}

The Supporting Information is available free of charge at https://pubs.acs.org/doi/10.1021/jacs.1c07067.

Experimental procedures, additional information, and analytical data for all new compounds (PDF)

\section{Accession Codes}

CCDC 2043273 and 2091821-2091822 contain the supplementary crystallographic data for this paper. These data can be obtained free of charge via www.ccdc.cam.ac.uk/data request/ cif, or by emailing data_request@ccdc.cam.ac.uk, or by contacting The Cambridge Crystallographic Data Centre, 12 Union Road, Cambridge CB2 1EZ, UK; fax: +44 1223336033.

\section{AUTHOR INFORMATION}

\section{Corresponding Author}

Benjamin List - Max-Planck-Institut für Kohlenforschung, 45470 Mülheim an der Ruhr, Germany; 이잉o.org/00000002-9804-599X; Email: list@kofo.mpg.de

\section{Authors \\ Sebastian A. Schwengers - Max-Planck-Institut für Kohlenforschung, 45470 Mülheim an der Ruhr, Germany \\ Chandra Kanta De - Max-Planck-Institut für Kohlenforschung, 45470 Mülheim an der Ruhr, Germany \\ Oleg Grossmann - Max-Planck-Institut für Kohlenforschung, 45470 Mülheim an der Ruhr, Germany \\ Joyce A. A. Grimm - Max-Planck-Institut für Kohlenforschung, 45470 Mülheim an der Ruhr, Germany \\ Natascha R. Sadlowski - Max-Planck-Institut für Kohlenforschung, 45470 Mülheim an der Ruhr, Germany \\ Gabriela G. Gerosa - Max-Planck-Institut für Kohlenforschung, 45470 Mülheim an der Ruhr, Germany}

Complete contact information is available at:

https://pubs.acs.org/10.1021/jacs.1c07067

\section{Funding}

Open access funded by Max Planck Society.

\section{Notes}

The authors declare the following competing financial interest(s): The authors filed a patent on the utilization of hexachlorophosphazonium salts toward the synthesis of imidodiphosphoryl-derived catalysts.

\section{ACKNOWLEDGMENTS}

Generous support from the Deutsche Forschungsgemeinschaft (Leibniz Award to B.L. and Cluster of Excellence Ruhr Explores Solvation, RESOLV), and the European Research Council (ERC, European Union's Horizon 2020 research and innovation program " $\mathrm{C}-\mathrm{H}$ Acids for Organic Synthesis, CHAOS” Advanced Grant Agreement No. 694228) is gratefully acknowledged. We also thank the technicians of our group, the MS department, Nils Nöthling and Dr. Richard Goddard from the X-ray department, Dr. Markus Leutzsch from our NMR department, and Veronika Dietl from the chromatography department for their excellent service. We also acknowledge DESY (Hamburg, Germany), a member of the Helmholtz Association HGF, for the provision of experimental facilities. Parts of this research were carried out at PETRA III, and we would like to thank Sofiane Saouane and Eva Crosas for assistance in using the P11 Bio-Imaging and Diffraction Beamline.

\section{REFERENCES}

(1) (a) Arpe, H.-J. Industrielle Organische Chemie; Wiley-VCH: Weinheim, Germany, 2007; Vol. 6; pp 1-536. (b) Yamamoto, H.; Ishihara, K. Acid Catalysis in Modern Organic Synthesis; Wiley-VCH: Weinheim, Germany, 2008; pp 1-1112. (c) Busca, G. Acid Catalysts in Industrial Hydrocarbon Chemistry. Chem. Rev. 2007, 107, 53665410.

(2) List, B.; Lerner, R. A.; Barbas, C. F. Proline-Catalyzed Direct Asymmetric Aldol Reactions. J. Am. Chem. Soc. 2000, 122, 23952396.

(3) (a) Akiyama, T.; Itoh, J.; Yokota, K.; Fuchibe, K. Enantioselective Mannich-Type Reaction Catalyzed by a Chiral Brønsted Acid. Angew. Chem., Int. Ed. 2004, 43, 1566-1568. (b) Uraguchi, D.; Terada, M. Chiral Brønsted Acid-Catalyzed Direct Mannich Reactions via Electrophilic Activation. J. Am. Chem. Soc. 2004, 126, 5356-5357. (c) Hatano, M.; Moriyama, K.; Maki, T.; Ishihara, K. Which is the Actual Catalyst: Chiral Phosphoric Acid or Chiral Calcium Phosphate? Angew. Chem., Int. Ed. 2010, 49, 38233826.

(4) Selected examples of chiral Brønsted acids: (a) Nakashima, D.; Yamamoto, H. Design of Chiral N-Triflyl Phosphoramide as a Strong Chiral Brønsted Acid and Its Application to Asymmetric Diels-Alder Reaction. J. Am. Chem. Soc. 2006, 128, 9626-9627. (b) Berkessel, A.; Christ, P.; Leconte, N.; Neudörfl, J.-M.; Schäfer, M. Synthesis and Structural Characterization of a New Class of Strong Chiral Brønsted Acids: 1,1'-Binaphthyl-2,2'-bis(sulfuryl)imides (JINGLEs). Eur. J. Org. Chem. 2010, 2010, 5165-5170. (c) Garcia-Garcia, P.; Lay, F.; Garcia-Garcia, P.; Rabalakos, C.; List, B. A Powerful Chiral Counteranion Motif for Asymmetric Catalysis. Angew. Chem., Int. Ed. 2009, 48, 4363-4366. (d) Kampen, D.; Ladépêche, A.; Claßen, G.; List, B. Brønsted Acid-Catalyzed Three-Component HosomiSakurai Reactions. Adv. Synth. Catal. 2008, 350, 962-966. (e) List, B.; Kaib, P. Highly Acidic BINOL-Derived Phosphoramidimidates and their Application in the Brønsted Acid Catalyzed Synthesis of $\alpha$ Tocopherol. Synlett 2015, 27, 156-158. (f) Vellalath, S.; Coric, I.; List, B. N-Phosphinyl Phosphoramide-A Chiral Brønsted Acid Motif for the Direct Asymmetric N,O-Acetalization of Aldehydes. Angew. Chem. 2010, 122, 9943-9946. (g) Wakchaure, V. N.; List, B. A New Structural Motif for Bifunctional Brønsted Acid/Base Organocatalysis. Angew. Chem., Int. Ed. 2010, 49, 4136-4139. 
(5) (a) Mayer, S.; List, B. Asymmetric Counteranion-Directed Catalysis. Angew. Chem., Int. Ed. 2006, 45, 4193-4195. (b) Mahlau, M.; List, B. Asymmetric Counteranion-Directed Catalysis: Concept, Definition, and Applications. Angew. Chem., Int. Ed. 2013, 52, 518533. (c) Hamilton, G. L.; Kang, E. J.; Mba, M.; Toste, F. D. A Powerful Chiral Counterion Strategy for Asymmetric Transition Metal Catalysis. Science 2007, 317, 496-499.

(6) (a) Akiyama, T.; Mori, K. Stronger Brønsted Acids: Recent Progress. Chem. Rev. 2015, 115, 9277-9306. (b) Kaupmees, K.; Tolstoluzhsky, N.; Raja, S.; Rueping, M.; Leito, I. On the acidity and reactivity of highly effective chiral Brønsted acid catalysts: establishment of an acidity scale. Angew. Chem., Int. Ed. 2013, 52, 1156911572. (c) Cheon, C. H.; Yamamoto, H. Super Brønsted acid catalysis. Chem. Commun. 2011, 47, 3043-3056.

(7) Coric, I.; List, B. Asymmetric spiroacetalization catalysed by confined Brønsted acids. Nature 2012, 483, 315-319.

(8) (a) An, D.; Fan, Y.-S.; Gao, Y.; Zhu, Z.-Q.; Zheng, L.-Y.; Zhang, S.-Q. Highly Enantioselective Biginelli Reaction Catalyzed by Double Axially Chiral Bisphosphorylimides. Eur. J. Org. Chem. 2014, 2014, 301-306. (b) Chen, Y.-Y.; Jiang, Y.-J.; Fan, Y.-S.; Sha, D.; Wang, Q.; Zhang, G.; Zheng, L.; Zhang, S. Double axially chiral bisphosphorylimides as novel Brønsted acids in asymmetric three-component Mannich reaction. Tetrahedron: Asymmetry 2012, 23, 904-909. (c) Das, S.; Liu, L.; Zheng, Y.; Alachraf, M. W.; Thiel, W.; De, C. K.; List, B. Nitrated Confined Imidodiphosphates Enable a Catalytic Asymmetric Oxa-Pictet-Spengler Reaction. J. Am. Chem. Soc. 2016, 138, 9429-9432. (d) Gualtierotti, J. B.; Pasche, D.; Wang, Q.; Zhu, J. Phosphoric Acid Catalyzed Desymmetrization of Bicyclic Bislactones Bearing an All-Carbon Stereogenic Center: Total Syntheses of (-)-Rhazinilam and (-)-Leucomidine B. Angew. Chem., Int. Ed. 2014, 53, 9926-9930. (e) Jindal, G.; Sunoj, R. B. Axially Chiral Imidodiphosphoric Acid Catalyst for Asymmetric Sulfoxidation Reaction: Insights on Asymmetric Induction. Angew. Chem., Int. Ed. 2014, 53, 4432-4436. (f) Kim, J. H.; Coric, I.; Palumbo, C.; List, B. Resolution of Diols via Catalytic Asymmetric Acetalization. J. Am. Chem. Soc. 2015, 137, 1778-1781. (g) Kim, J. H.; Coric, I.; Vellalath, S.; List, B. The Catalytic Asymmetric Acetalization. Angew. Chem., Int. Ed. 2013, 52, 4474-4477. (h) Li, J.-Y.; Kim, H. Y.; Oh, K. Enantiodivergent Brucine Diol-Catalyzed 1,3-Dipolar Cycloaddition of Azomethine Ylides with $\alpha, \beta$-Unsaturated Ketones. Adv. Synth. Catal. 2016, 358, 984-993. (i) Li, X. Y.; Hu, W. T.; Xiong, Q. J.; Ye, S. M.; Huang, Y. M.; Guo, Q. X. Chiral Anion-Induced Catalytic Asymmetric Direct Dehydrative Coupling of 3-Vinylindoles and 3Indolylmethanols. Adv. Synth. Catal. 2019, 361, 1803-1807. (j) Li, Y.; Wan, M.; Sun, S.; Fu, Z.; Huang, H.; Liu, L. Efficient access to chiral benzo[c]chromenes via asymmetric transfer hydrogenation of ketals. Org. Chem. Front. 2018, 5, 1280-1283. (k) List, B.; Kim, J.; Tap, A.; Liu, L. Catalytic Asymmetric Thioacetalization of Aldehydes. Synlett 2017, 28, 333-336. (1) Liu, L.; Leutzsch, M.; Zheng, Y.; Alachraf, M. W.; Thiel, W.; List, B. Confined Acid-Catalyzed Asymmetric Carbonyl-Ene Cyclization. J. Am. Chem. Soc. 2015, 137, 1326813271. (m) Morse, P. D.; Nguyen, T. M.; Cruz, C. L.; Nicewicz, D. A. Enantioselective counter-anions in photoredox catalysis: the asymmetric cation radical Diels-Alder reaction. Tetrahedron 2018, 74, 3266-3272. (n) Tsui, G. C.; Liu, L.; List, B. The Organocatalytic Asymmetric Prins Cyclization. Angew. Chem., Int. Ed. 2015, 54, 77037706. (o) Wan, M.; Sun, S.; Li, Y.; Liu, L. Organocatalytic Redox Deracemization of Cyclic Benzylic Ethers Enabled by An Acetal Pool Strategy. Angew. Chem., Int. Ed. 2017, 56, 5116-5120. (p) Wu, K.; Jiang, Y. J.; Fan, Y. S.; Sha, D.; Zhang, S. Double Axially Chiral Bisphosphorylimides Catalyzed Highly Enantioselective and Efficient Friedel-Crafts Reaction of Indoles with Imines. Chem. - Eur. J. 2013, 19, 474-478. (q) Xie, Y.; Cheng, G. J.; Lee, S.; Kaib, P. S.; Thiel, W.; List, B. Catalytic Asymmetric Vinylogous Prins Cyclization: A Highly Diastereo- and Enantioselective Entry to Tetrahydrofurans. J. Am. Chem. Soc. 2016, 138, 14538-14541. (r) Xie, Y.; List, B. Catalytic Asymmetric Intramolecular $[4+2]$ Cycloaddition of In Situ Generated ortho-Quinone Methides. Angew. Chem., Int. Ed. 2017, 56, 4936-4940. (s) Zhuo, M. H.; Jiang, Y. J.; Fan, Y. S.; Gao, Y.; Liu,
S.; Zhang, S. Enantioselective Synthesis of Triarylmethanes by Chiral Imidodiphosphoric Acids Catalyzed Friedel-Crafts Reactions. Org. Lett. 2014, 16, 1096-1099.

(9) Liu, L.; Kaib, P. S.; Tap, A.; List, B. A General Catalytic Asymmetric Prins Cyclization. J. Am. Chem. Soc. 2016, 138, 1082210825.

(10) Kaib, P. S.; Schreyer, L.; Lee, S.; Properzi, R.; List, B. Extremely Active Organocatalysts Enable a Highly Enantioselective Addition of Allyltrimethylsilane to Aldehydes. Angew. Chem., Int. Ed. 2016, 55, 13200-13203.

(11) Mitschke, B.; Turberg, M.; List, B. Confinement as a Unifying Element in Selective Catalysis. Chem. 2020, 6, 2515-2532.

(12) Schreyer, L.; Properzi, R.; List, B. IDPi Catalysis. Angew. Chem., Int. Ed. 2019, 58, 12761-12777.

(13) Tsuji, N.; Kennemur, J. L.; Buyck, T.; Lee, S.; Prévost, S.; Kaib, P. S. J.; Bykov, D.; Farès, C.; List, B. Activation of olefins via asymmetric Brønsted acid catalysis. Science 2018, 359, 1501-1505.

(14) Schreyer, L.; Kaib, P. S. J.; Wakchaure, V. N.; Obradors, C.; Properzi, R.; Lee, S.; List, B. Confined acids catalyze asymmetric single aldolizations of acetaldehyde enolates. Science 2018, 362, 216219.

(15) Bae, H. Y.; Höfler, D.; Kaib, P. S. J.; Kasaplar, P.; De, C. K.; Döhring, A.; Lee, S.; Kaupmees, K.; Leito, I.; List, B. Approaching sub-ppm-level asymmetric organocatalysis of a highly challenging and scalable carbon-carbon bond forming reaction. Nat. Chem. 2018, 10, 888-894.

(16) Properzi, R.; Kaib, P. S. J.; Leutzsch, M.; Pupo, G.; Mitra, R.; De, C. K.; Song, L.; Schreiner, P. R.; List, B. Catalytic enantiocontrol over a non-classical carbocation. Nat. Chem. 2020, 12, 1174-1179.

(17) List, B.; Lee, S.; Kaib, P. N-Triflylphosphorimidoyl Trichloride: A Versatile Reagent for the Synthesis of Strong Chiral Brønsted Acids. Synlett 2017, 28, 1478-1480.

(18) Phipps, R. J.; Hamilton, G. L.; Toste, F. D. The progression of chiral anions from concepts to applications in asymmetric catalysis. Nat. Chem. 2012, 4, 603-614.

(19) Becke-Goehring, M.; Lehr, W. Ein neues Phosphornitridchlorid, $\mathrm{P}_{3} \mathrm{NCl}_{12}$. Chem. Ber. 1961, 94, 1591-1594.

(20) Alternative synthesis of HCPP: (a) Rivard, E.; Lough, A. J.; Manners, I. A New, Convenient Synthesis of the Linear Phosphazene Salt $\left[\mathrm{Cl}_{3} \mathrm{P}=\mathrm{N}=\mathrm{PCl}_{3}\right] \mathrm{Cl}$ : Preparation of Higher Linear Homologues $\left[\mathrm{Cl}_{3} \mathrm{P}=\mathrm{N}-\left(\mathrm{PCl}_{2}=\mathrm{N}\right)_{\mathrm{x}}=\mathrm{PCl}_{3}\right] \mathrm{Cl}(\mathrm{x}=1-3)$ and the 16-Membered Macrocycle $\left[\mathrm{NCCl}\left(\mathrm{NPCl}_{2}\right)_{3}\right]_{2}$. Inorg. Chem. 2004, 43, 2765-2767. (b) Wang, B.; Rivard, E.; Manners, I. A New High-Yield Synthesis of $\mathrm{Cl}_{3} \mathrm{P}=\mathrm{NSiMe}_{3}$, a Monomeric Precursor for the Controlled Preparation of High Molecular Weight Polyphosphazenes. Inorg. Chem. 2002, 41, 1690-1691.

(21) Liao, S.; Coric, I.; Wang, Q.; List, B. Activation of $\mathrm{H}_{2} \mathrm{O}_{2}$ by Chiral Confined Brønsted Acids: a Highly Enantioselective Catalytic Sulfoxidation. J. Am. Chem. Soc. 2012, 134, 10765-10768.

(22) (a) Llera, J. M.; Fernández, I.; Alcudia, F. An Efficient Synthesis of Both Enantiomers of Chiral non Racemic Methylsulfoxides from DAG. Tetrahedron Lett. 1991, 32, 7299-7302. (b) Katopodis, A. G.; Smith, H. A.; May, S. W. New Oxyfunctionalization Capabilities for $\omega$-Hydroxylases: Asymmetric Aliphatic Sulfoxidation and Branched Ether Demethylation. J. Am. Chem. Soc. 1988, 110, 897-899. (c) Glahsl, G.; Herrmann, R. (+)-(3-Oxocamphorsulphonyl)oxaziridine as a Highly Stereoselective Reagent for the Oxidation of Sulphides to Chiral Sulfoxides. J. Chem. Soc., Perkin Trans. 1 1988, 1753-1757. (d) Drabowicz, J.; Mikolajczyk, M. Asymmetric Oxidation of Sulfides to Sulfoxides Catalyzed by $\beta$-Cyclodextrin. Phosphorus Sulfur Relat. Elem. 1984, 21, 245-248.

(23) (a) Becker, C. L.; Engstrom, K. M.; Kerdesky, F. A.; Tolle, J. C.; Wagaw, S. H.; Wang, W. A Convergent Process for the Preparation of Adamantane 11- $\beta$-HSD-1 Inhibitors. Org. Process Res. Dev. 2008, 12, 1114-1118. (b) Qiao, K.; Yokoyama, C. Koch carbonylation of tertiary alcohols in the presence of acidic ionic liquids. Catal. Commun. 2006, 7, 450-453. (c) Farooq, O.; Marcelli, M.; Prakash, G. K. S.; Olah, G. A. Superacid-Catalyzed Electrophilic Formylation of Adamantane with Carbon Monoxide Competing with 
Koch-Haaf Carboxylation. J. Am. Chem. Soc. 1988, 110, 864-867. (d) Rueping, M.; Nachtsheim, B. J. A review of new developments in the Friedel-Crafts alkylation - From green chemistry to asymmetric catalysis. Beilstein J. Org. Chem. 2010, 6.

(24) Riddlestone, I. M.; Kraft, A.; Schaefer, J.; Krossing, I. Taming the Cationic Beast: Novel Developments in the Synthesis and Application of Weakly Coordinating Anions. Angew. Chem., Int. Ed. 2018, 57, 13982-14024.

(25) Koppel, I. A.; Taft, R. W.; Anvia, F.; Zhu, S.-Z.; Hu, L.-Q.; Sung, K.-S.; DesMarteau, D. D.; Yagupolskii, L. M.; Yagupolskii, Y. L. The Gas-Phase Acidities of Very Strong Neutral Brønsted Acids. J. Am. Chem. Soc. 1994, 116, 3047-3057.

(26) (a) Yagupolskii, L. M.; Petrik, V. N.; Kondratenko, N. V.; Sooväli, L.; Kaljurand, I.; Leito, I.; Koppel, I. A. The immense acidifying effect of the supersubstituent $=\mathrm{NSO}_{2} \mathrm{CF}_{3}$ on the acidity of amides and amidines of benzoic acids in acetonitrile. J. Chem. Soc., Perkin Trans. 2 2002, 1950-1955. (b) Yagupolskii, L. M.; Garlyauskajte, R. Y.; Kondratenko, N. V. N(Trifluoromethylsulfonyl)arenesulfonimidoyl and $N, N^{\prime}$-Bis(trifluoromethylsulfonyl)arenesulfonodiimidoyl Chlorides. Synthesis 1992, 1992, 749-750. (c) Yagupolskii, Y. L.; Bezdudnyi, A. V.; Yagupolskii, L. M. N-(Trifluoromethylsulfonyl)trifluoromethanesulfinimidic acid and its salts. J. Fluorine Chem. 2002, 115, 129-132. (d) Posternak, A. G.; Garlyauskayte, R. Y.; Polovinko, V. V.; Yagupolskii, L. M.; Yagupolskii, Y. L. New kinds of organic superacids. Bis (perfluoroalkylsulfonylimino)trifluoromethanesulfonic acids and their trimethylsilyl esters. Org. Biomol. Chem. 2009, 7, 1642-1645. (e) Posternak, A. G.; Garlyauskayte, R. Y.; Yagupolskii, L. M. A novel Brønsted acid catalyst for Friedel-Crafts acylation. Tetrahedron Lett. 2009, 50, 446447. (f) Garlyauskayte, R. Y.; Chernega, A. N.; Michot, C.; Armand, M.; Yagupolskii, Y. L.; Yagupolskii, L. M. Synthesis of new organic super acids- $\mathrm{N}$-(trifluoromethylsulfonyl)imino derivatives of trifluoromethanesulfonic acid and bis(trifluoromethylsulfonyl)imide. Org. Biomol. Chem. 2005, 3, 2239-2243.

(27) Kutt, A.; Rodima, T.; Saame, J.; Raamat, E.; Maemets, V.; Kaljurand, I.; Koppel, I. A.; Garlyauskayte, R. Y.; Yagupolskii, Y. L.; Yagupolskii, L. M.; Bernhardt, E.; Willner, H.; Leito, I. Equilibrium Acidities of Superacids. J. Org. Chem. 2011, 76, 391-395.

(28) Koppel, I.; Koppel, J.; Maria, P.-C.; Gal, J.-F.; Notario, R.; Vlasov, V. M.; Taft, R. W. Comparison of Brønsted acidities of neutral $\mathrm{NH}$-acids in gas phase, dimethyl sulfoxide and water. Int. J. Mass Spectrom. Ion Processes 1998, 175, 61-69.

(29) Koppel, I. A.; Koppel, J.; Leito, I.; Koppel, I.; Mishima, M.; Yagupolskii, L. M. The enormous acidifying effect of the supersubstituent $=\mathrm{NSO}_{2} \mathrm{CF}_{3}$ on the acidity of derivatives of benzenesulfonamide and toluene-p-sulfonamide in the gas phase and in dimethyl sulfoxide. J. Chem. Soc., Perkin Trans. 2 2001, 229-232.

(30) Garlyauskajte, R. Y.; Sereda, S. V.; Yagupolskii, L. M. The Unusual Reactivity of the Mono- and Bis- N-(Trifluoromethylsulfonyl)-Substituted Azaanalogs of Arenesulfonochlorides. Tetrahedron 1994, 50, 6891-6906.

(31) (a) Mayer, U.; Gutmann, V.; Gerger, W. The Acceptor Number - A Quantitative Empirical Parameter for the Electrophilic Properties of Solvents. Monatsh. Chem. 1975, 106, 1235-1257. (b) Beckett, M. A.; Strickland, G. C.; Holland, J. R.; Sukumar Varma, K. A convenient n.m.r. method for the measurement of Lewis acidity at boron centres: correlation of reaction rates of Lewis acid initiated epoxide polymerizations with Lewis acidity. Polymer 1996, 37, 4629-4631. (c) Gutmann, V. Solvent Effects on the Reactivities of Organometallic Compounds. Coord. Chem. Rev. 1976, 18, 225-255.

(32) (a) Klare, H. F. T.; Albers, L.; Süsse, L.; Keess, S.; Müller, T.; Oestreich, M. Silylium Ions: From Elusive Reactive Intermediates to Potent Catalysts. Chem. Rev. 2021, 121, 5889-5985. (b) Höfler, D.; van Gemmeren, M.; Wedemann, P.; Kaupmees, K.; Leito, I.; Leutzsch, M.; Lingnau, J. B.; List, B. 1,1,3,3-Tetratriflylpropene (TTP): A Strong, Allylic C-H Acid for Brønsted and Lewis Acid Catalysis. Angew. Chem., Int. Ed. 2017, 56, 1411-1415.
(33) (a) Müther, K.; Hrobárik, P.; Hrobáriková, V.; Kaupp, M.; Oestreich, M. The Family of Ferrocene-Stabilized Silylium Ions: Synthesis, ${ }^{29} \mathrm{Si}$ NMR Characterization, Lewis Acidity, Substituent Scrambling, and Quantum-Chemical Analyses. Chem. - Eur. J. 2013, 19, 16579-16594. (b) Walker, J. C. L.; Klare, H. F. T.; Oestreich, M. Cationic silicon Lewis acids in catalysis. Nat. Rev. Chem. 2020, 4, 5462. (c) Großekappenberg, H.; Reißmann, M.; Schmidtmann, M.; Müller, T. Quantitative Assessment of the Lewis Acidity of Silylium Ions. Organometallics 2015, 34, 4952-4958.

(34) Caputo, C. B.; Hounjet, L. J.; Dobrovetsky, R.; Stephan, D. W. Lewis Acidity of Organofluorophosphonium Salts: Hydrodefluorination by a Saturated Acceptor. Science 2013, 341, 1374-1377.

(35) (a) Pérez, M.; Hounjet, L. J.; Caputo, C. B.; Dobrovetsky, R.; Stephan, D. W. Olefin isomerization and hydrosilylation catalysis by Lewis acidic organofluorophosphonium salts. J. Am. Chem. Soc. 2013, 135, 18308-18310. (b) Vogler, M.; Süsse, L.; LaFortune, J. H. W.; Stephan, D. W.; Oestreich, M. Electrophilic Phosphonium Cations as Lewis Acid Catalysts in Diels-Alder Reactions and Nazarov Cyclizations. Organometallics 2018, 37, 3303-3313. 